\title{
Matter Radii of Light Halo Nuclei
}

\author{
J. S. Al-Khalili and J. A. Tostevin \\ Department of Physics, University of Surrey, Guildford, Surrey, GU2 5XH, United Kingdom
}

(Received 16 February 1996)

\begin{abstract}
We reexamine the matter radii of diffuse halo nuclei, as deduced from reaction cross section measurements at high energy. Careful consideration is given to the intrinsic few-body structure of these projectiles and the adiabatic nature of the projectile-target interaction. Using ${ }^{11} \mathrm{Li},{ }^{11} \mathrm{Be}$, and ${ }^{8} \mathrm{~B}$ as examples we show that data require significantly larger matter radii than previously reported. The revised value for ${ }^{11} \mathrm{Li}$ of $3.55 \mathrm{fm}$ is consistent with three-body models with significant $1 s$-intruder state components, which reproduce experimental ${ }^{9} \mathrm{Li}$ momentum distributions following ${ }^{11} \mathrm{Li}$ breakup, but were hitherto thought to be at variance with cross section data. [S0031-9007(96)00289-X]
\end{abstract}

PACS numbers: 21.10.Gv, 11.80.Fv, 25.10.+s, 27.20.+n

Reaction cross section measurements at energies of several hundred $\mathrm{MeV} /$ nucleon have been used to study the radial extent of matter densities of short lived exotic nuclei produced by fragmentation [1,2]. Extensive tables of deduced radii are now available in the literature, e.g., [3]. Glauber theoretical methods [4,5] have been the basis for these assignments, and in particular the approximation $[3,5]$ in which it is assumed that the projectile and target nuclei present static density distributions [6] whose geometric overlap determines the reaction cross section. To high accuracy, the deduced rms radii are found to be essentially independent of the details of the projectile density distributions assumed, e.g., [3,7]. The accuracy of such deduced root mean square (rms) radii is of considerable importance since they are routinely used as empirical measures in constructing, constraining, and assessing theoretical models of halo structures for use in the interpretation of data.

At the heart of the static density model is the neglect of correlations between the projectile (and target) constituents, each projectile nucleon being assumed to carry the same single particle density [5]. This assumption would appear to work well for spatially localized nuclei such as ${ }^{12} \mathrm{C}[8]$. For weakly bound systems such as halo nuclei, however, the intrinsic few-body nature or granularity of the projectiles implies strong spatial correlations between the valence nucleons and the more localized core. At incident energies of order $800 \mathrm{MeV} /$ nucleon one must also consider the relevant time scales for a significant motion of these valence particles inside the projectile and that for the passage of the same particle through the target interaction region. In breakup studies narrow momentum widths are associated with these valence particles which have characteristic kinetic energies of order 10-40 MeV within the projectile [9]. For this reason reaction models $[10,11]$ make an adiabatic approximation, freezing the position coordinates of the few-body projectile constituents during the interaction. Physical observables are then obtained by suitably averaging the resulting position dependent reaction amplitudes over the relevant position probability distributions of these constituents.

This few-body picture suggests a quite different description of the projectile-target interaction and formulation of the reaction cross section. Consider, for example, ${ }^{11} \mathrm{Li}$ as a pair of neutrons bound to a ${ }^{9} \mathrm{Li}$ core. For an impact parameter $b$ of the ${ }^{11} \mathrm{Li}$ center of mass, Fig. 1, such that the projectile static density (shaded circle) overlaps the target, many spatial configurations of the constituent bodies will not overlap the target. The expectation is that the valence nucleon (large $b$ ) contribution to the reaction cross section will be reduced or, alternatively, that the collision will appear more transparent than otherwise expected. Nishioka and Johnson [12] investigated related adiabatic effects on light-ion composite projectile $\left(d, t,{ }^{3} \mathrm{He}\right.$, and $\left.\alpha\right)$ cross sections in the energy range $100 \leq E \leq 350 \mathrm{MeV} /$ nucleon. The effects were very significant for the extended deuteron but small for the $\alpha$ particle. Estimates of the accuracy of the static density model for ${ }^{11} \mathrm{Li}$ were considered earlier by Takigawa et al. [13] for a simplified two-body (di-neutron) halo density and at lower energies, where the adiabatic limit is expected to be less reliable. They demonstrated clearly the convergence of the two-body

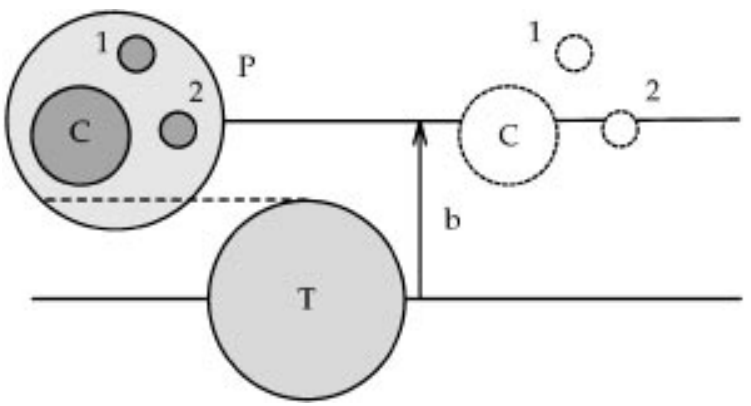

FIG. 1. Schematic representation of the static density (shaded circle) and few-body adiabatic (frozen coordinate) treatments of the three-body projectile $(P)$ and target $(T)$ collision at impact parameter $b$. In the spatial configuration drawn the few-body projectile does not overlap the target. 
and static density models in the limit of tight valence nucleon binding. They concluded that static density calculations would indeed overestimate reaction cross sections; however, the model used was too crude to allow a quantitative discussion. The overestimation of cross sections in the static density model was also recognized previously by Chulkov et al. [7].

In this Letter we examine the quantitative implications of the few-body adiabatic description for deduced matter radii of halo nuclei. We take as examples two-body ${ }^{8} \mathrm{~B}$ and ${ }^{11} \mathrm{Be}$ and three-body ${ }^{11} \mathrm{Li}$ systems for which cross section data are available for each composite and core $\left({ }^{7} \mathrm{Be},{ }^{10} \mathrm{Be}\right.$, and $\left.{ }^{9} \mathrm{Li}\right)$ nucleus on a ${ }^{12} \mathrm{C}$ target at $800 \mathrm{MeV} /$ nucleon. Data are also available for the nucleon- ${ }^{12} \mathrm{C}$ system, so that all projectile constituenttarget subsystems can be interrogated and compared with experiment. In common with the analysis of Takigawa et al. [13] we will apply the static density approximation to the spatially localized core-target and valence nucleontarget subsystems. Additionally, the adiabatic (frozen coordinate) treatment of these constituents allows us to study carefully the implications of a realistic treatment of the two- and three-body nature of the projectile wave functions on calculated cross sections.

In Glauber theory [4] the reaction cross section for projectile $P$ is

$$
\sigma_{R}(P)=2 \pi \int_{0}^{\infty} d b b\left[1-T_{P}(b)\right],
$$

where $T_{P}(b)$, the squared modulus of the Glauber $S$ matrix, is the transparency of the collision at impact parameter $b$ of the projectile center of mass. In the static density limit

$$
T_{P}^{\mathrm{SD}}(b)=\exp \left[-\bar{\sigma}_{N N}^{P T} \int d^{2} \mathbf{x} \rho_{P}^{(z)}(|\mathbf{x}|) \rho_{T}^{(z)}(|\mathbf{b}-\mathbf{x}|)\right],
$$

where $\bar{\sigma}_{N N}^{P T}$ is the free nucleon-nucleon $(N N)$ cross section, at the relevant energy, appropriate for the projectile and target [14] with densities $\rho_{P}$ and $\rho_{T}$, and the

$$
\rho_{i}^{(z)}(b)=\int_{-\infty}^{\infty} d z \rho_{i}\left(\sqrt{b^{2}+z^{2}}\right)
$$

are the $z$-integrated densities or thickness functions. Here only the projectile ground state density enters the calculation and few-body correlations, the granular nature of the projectile, do not enter explicitly.

In the few-body adiabatic limit, the transparency function is [15]

$$
T_{P}^{\mathrm{AD}}(b)=\left|\left\langle\Phi_{0}^{n}\left|S_{C}\left(b_{C}\right) S_{v}\left(b_{v}\right)\right| \Phi_{0}^{n}\right\rangle\right|^{2},
$$

where $\left|\Phi_{0}^{n}\right\rangle$ is the wave function for the relative motion of the $n$-constituent bodies in the projectile ground state, the bra-ket denoting integration over these internal coordinates. For a two-body (one valence nucleon + core) projectile the core-target and valence nucleon-target
$S$ matrices, in the static density limit, are

$$
\begin{aligned}
S_{C}\left(b_{C}\right) & =\left[T_{C}^{\mathrm{SD}}\left(b_{C}\right)\right]^{1 / 2}, \\
S_{v}\left(b_{v}\right) & \equiv S_{1}\left(b_{1}\right)=\left[T_{N}^{\mathrm{SD}}\left(b_{1}\right)\right]^{1 / 2},
\end{aligned}
$$

with $b_{C}$ the impact parameter of the core and $T_{N}^{\mathrm{SD}}$ the analog of Eq. (2) for the nucleon. For a three-body (two valence nucleon + core) system, then of course

$$
S_{v}\left(b_{v}\right) \equiv S_{1}\left(b_{1}\right) S_{2}\left(b_{2}\right),
$$

where the coordinates, in the plane perpendicular to the beam direction, are shown in Fig. 2. Equations (1) through (6) are calculated exactly in the following for realistic two- and three-body wave functions $\left|\Phi_{0}^{n}\right\rangle$. The explicit forms of the three-body wave function for ${ }^{11} \mathrm{Li}$ are given in [11].

We apply the formalism above to calculate reaction cross sections in the static density and adiabatic limits for the one- and two-neutron halo nuclei ${ }^{11} \mathrm{Be}$ and ${ }^{11} \mathrm{Li}$, and the one-proton halo nucleus candidate ${ }^{8} \mathrm{~B}$, all on a ${ }^{12} \mathrm{C}$ target at $800 \mathrm{MeV} /$ nucleon. The choice of energy and target was dictated by our wish to connect cross sections for all binary subsystems with experiment.

For all three incident nuclei, the static density calculations of the projectile-target $\left(T_{P}^{\mathrm{SD}}\right)$, core-target $\left(S_{C}\right)$, and valence particle-target $\left(S_{v}\right)$ subsystems use the prescription for $\bar{\sigma}_{N N}^{i T}(i=P, C, N)$ of Charagi and Gupta [14]. A Gaussian matter distribution is assumed for ${ }^{12} \mathrm{C}$ in all cases with rms matter radius $\left\langle r^{2}\right\rangle_{12}^{1 / 2}=2.32 \mathrm{fm} \mathrm{[3]}$. With these inputs, and assuming Gaussian matter distributions for the core nuclei with radii $\left\langle r^{2}\right\rangle_{9}^{1 / 2}=2.30 \mathrm{fm}$, $\left\langle r^{2}\right\rangle_{10}^{1 / 2}=2.28 \mathrm{fm}$, and $\left\langle r^{2}\right\rangle_{7}^{1 / 2}=2.31 \mathrm{fm}$, we calculate reaction cross sections for the core-target subsystems $\sigma_{R}\left({ }^{9} \mathrm{Li}\right)=796(796 \pm 6) \mathrm{mb}, \sigma_{R}\left({ }^{10} \mathrm{Be}\right)=813(813 \pm$ 10) $\mathrm{mb}$, and $\sigma_{R}\left({ }^{7} \mathrm{Be}\right)=738(738 \pm 9) \mathrm{mb}$. The empirical values, in parentheses, are taken from [2]. The deduced core radii agree with those of [3] within error bars. The calculated nucleon- ${ }^{12} \mathrm{C}$ cross section at $800 \mathrm{MeV}$ is

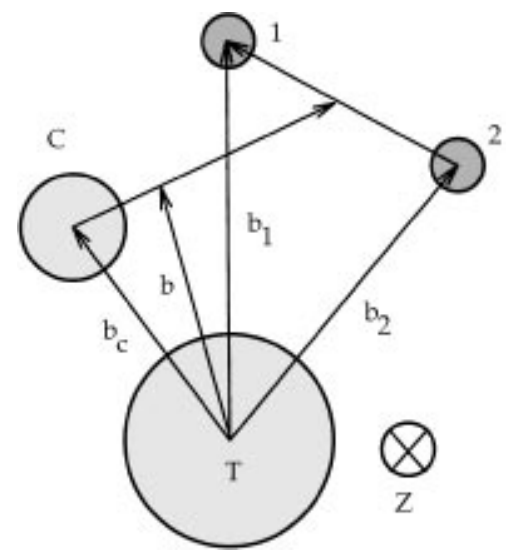

FIG. 2. Definition of position coordinates, in the plane perpendicular to the beam direction, in the case of a three-body (two valence nucleon + core) projectile. 
$\sigma_{R}(N)=231 \mathrm{mb}$ which also agrees with experiment [16] within quoted errors. Thus each projectile constituenttarget input to the few-body calculations, $S_{C}$ and $S_{v}$, is consistent with independent empirical data for that binary system.

Figure 3(a) shows the results of the static density and adiabatic calculations for the ${ }^{11} \mathrm{Li}+{ }^{12} \mathrm{C}$ system for a number of theoretical three-body wave functions of ${ }^{11} \mathrm{Li}$. We show the calculated cross sections versus the matter rms radius calculated from the wave function models. The horizontal band shows the experimental interaction cross section datum $\sigma\left({ }^{11} \mathrm{Li}\right)=1060 \pm 10 \mathrm{mb}$ [17] and
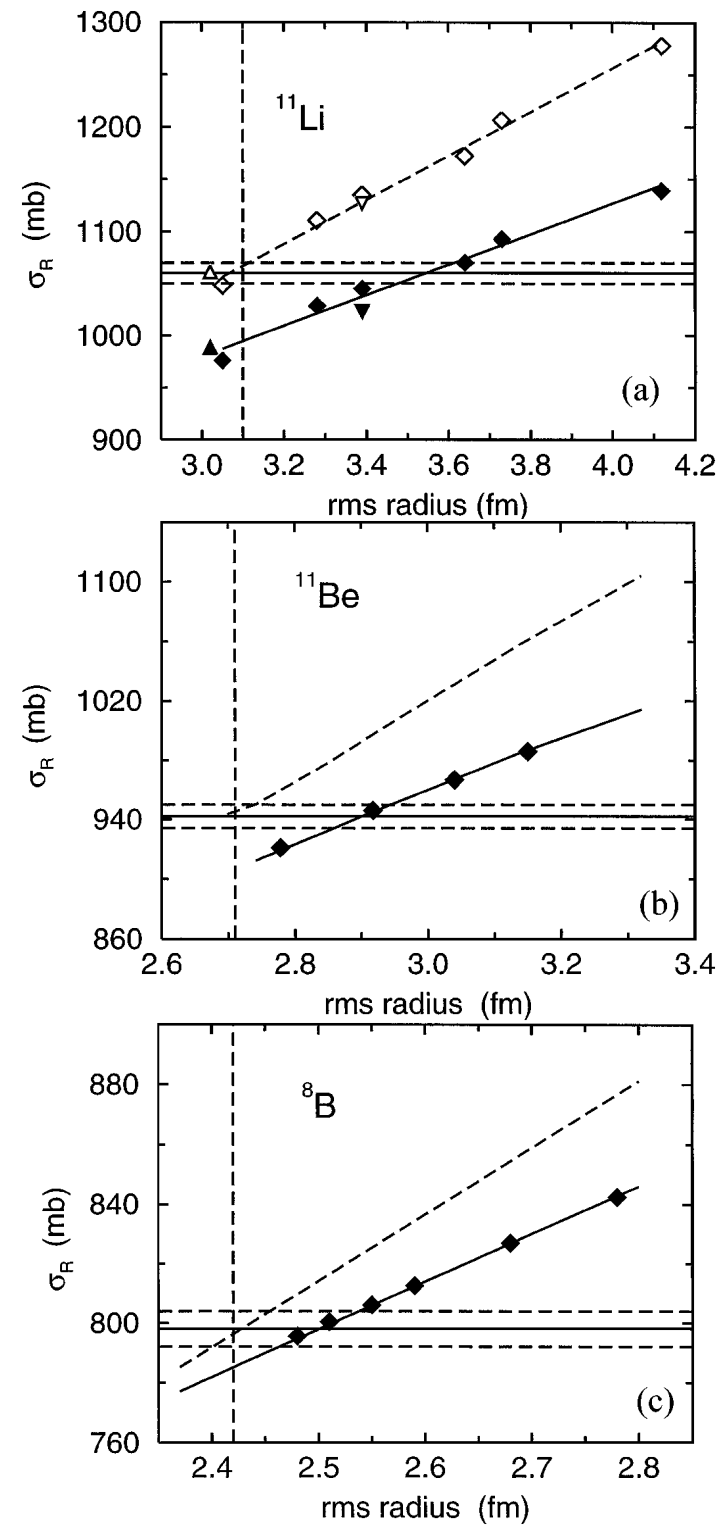

FIG. 3. Calculated static density and few-body adiabatic reaction cross sections at $800 \mathrm{MeV} /$ nucleon incident energy as a function of projectile $\mathrm{rms}$ matter radius, for a ${ }^{12} \mathrm{C}$ target. Parts (a), (b), and (c) of the figure are for ${ }^{11} \mathrm{Li},{ }^{11} \mathrm{Be}$, and ${ }^{8} \mathrm{~B}$ projectiles, respectively. Details are given in the text. the vertical dashed line the previously quoted matter radius $\left\langle r^{2}\right\rangle_{11}^{1 / 2}=3.10 \pm 0.17 \mathrm{fm} \mathrm{[3].}$

The (upper) open symbols are the results of the static density model and the (lower) full symbols those of the adiabatic calculations for each wave function model. The reduction in the calculated cross sections, or increased transparency of the projectile in the latter case, is immediately evident. From left to right the diamond symbols correspond to the P0 through P4 intruder $s$ wave (Faddeev) model wave functions of Thompson and Zhukov [18], with increasing rms radius. The extreme right hand point is a continuation of these model wave functions (P5) with a $1 s$-state scattering length of $-44 \mathrm{fm}$ and $80 \%\left(1 s_{1 / 2}\right)^{2}$ probability. The upright and inverted triangles are calculations using the L6A pairing model wave function [19], which in the static density picture fits the published radial value, and the weak binding potential $0 s$-wave intruder wave function (G1 of [18]) inspired by the work of Johannsen, Jensen, and Hansen [20]. The straight lines through these model points are to guide the eye.

The results of these calculations are indeed dramatic. Whereas static density calculations suggest a matter rms radius of order $3.1 \mathrm{fm}$, as reported previously, a correct treatment of the ${ }^{11} \mathrm{Li}$ three-body character now suggests the halo is very much more extended and that $\left\langle r^{2}\right\rangle_{11}^{1 / 2}=3.55 \pm 0.10 \mathrm{fm}$, firmly in the middle of the range of values generated by intruder state models which successfully reproduce empirical breakup momentum distributions [18].

Figure 3(b) shows the results of similar calculations but for the one-neutron halo system ${ }^{11} \mathrm{Be}$. Again the horizontal band shows the experimental cross section datum $\sigma\left({ }^{11} \mathrm{Be}\right)=942 \pm 8 \mathrm{mb}$ [2] and the vertical dashed line the previously reported rms matter radius $\left\langle r^{2}\right\rangle_{11}^{1 / 2}=2.71 \pm 0.05 \mathrm{fm}$ [3]. The results are qualitatively very similar to those of the three-body ${ }^{11} \mathrm{Li}$ case. The angled dashed line shows the static density calculations and the angled solid line and full symbols the adiabatic model results. In this case these lines connect a large number of calculations using simple two-body $\left(1 s_{1 / 2}\right)$ cluster wave functions for ${ }^{11} \mathrm{Be}$ using binding potentials with a range of geometries and with depth adjusted to reproduce the single neutron separation energy $0.503 \mathrm{MeV}$. The solid symbols are the results of adiabatic calculations for ${ }^{11} \mathrm{Be}$ wave functions [21] which include the effects of core $\left({ }^{10} \mathrm{Be}\right)$ deformation and excitation. The wave function with rms radius $2.92 \mathrm{fm}$, whose calculated cross section lies within experimental error bars, best reproduces the excited state spectrum of ${ }^{11} \mathrm{Be}$. These wave functions generate cross sections which follow precisely the trend of the inert core calculations and suggest a revised matter rms radius of $\left\langle r^{2}\right\rangle_{11}^{1 / 2}=2.90 \pm 0.05 \mathrm{fm}$.

Finally, in Fig. 3(c) we consider the one proton-halo nucleus candidate ${ }^{8} \mathrm{~B}$. The previously reported value of 
$\left\langle r^{2}\right\rangle_{8}^{1 / 2}=2.39 \pm 0.04 \mathrm{fm} \mathrm{[3]} \mathrm{was} \mathrm{very} \mathrm{close} \mathrm{to} \mathrm{that} \mathrm{for}$ ${ }^{7} \mathrm{Be},\left\langle r^{2}\right\rangle_{7}^{1 / 2}=2.33 \pm 0.02 \mathrm{fm}$ [3] suggesting, in spite of the very small proton separation energy $(0.137 \mathrm{MeV})$ that the last proton had rather limited extension. The experimental cross section for ${ }^{8} \mathrm{~B}$ has recently been revised to $\sigma\left({ }^{8} \mathrm{~B}\right)=798 \pm 6 \mathrm{mb}$ [22] and is shown by the horizontal band on the figure. Using the static density model and a Gaussian density, in the manner of [3], we obtain a revised static density estimate of $\left\langle r^{2}\right\rangle_{8}^{1 / 2}=2.42 \pm 0.03 \mathrm{fm}$, shown by the vertical dashed line. The angled dashed and solid lines are the results of static density and adiabatic model calculations for a large number of two-body $\left(0 p_{3 / 2}\right)$ cluster wave functions for ${ }^{8} \mathrm{~B}$ based on Woods-Saxon potential geometries. The diamonds use wave functions based on the often used cosh form cluster model interaction [23] and lie on the same lines. Although the differences between the model calculations are smaller than in the neutron halo cases, they remain very significant and suggest the rms radius of ${ }^{8} \mathrm{~B}$ should be revised to $\left\langle r^{2}\right\rangle_{8}^{1 / 2}=2.50 \pm 0.04 \mathrm{fm}$, indicating quite significant extension of the last proton distribution beyond that of the core.

In summary, we have reanalyzed experimental data of reaction cross sections for ${ }^{11} \mathrm{Be},{ }^{11} \mathrm{Li}$, and ${ }^{8} \mathrm{~B}$ projectiles on a ${ }^{12} \mathrm{C}$ target at $800 \mathrm{MeV} /$ nucleon using an adiabatic treatment of the internal coordinates of the two- and threebody projectiles. We verify that all binary channel inputs to the adiabatic model are consistent with the available experimental data for these independent systems. The granular structure of the projectiles implied by realistic few-body wave functions is shown to reduce considerably the calculated reaction cross sections and increase significantly the values of matter rms radii deduced from data when compared to static density estimates.

We deduce matter rms radii for ${ }^{11} \mathrm{Li},{ }^{11} \mathrm{Be}$, and ${ }^{8} \mathrm{~B}$ of $3.55 \pm 0.10,2.90 \pm 0.05$, and $2.50 \pm 0.04 \mathrm{fm}$, respectively, representing increases of $14.5 \%, 7 \%$, and $4.6 \%$ over previously tabulated values. Our revised radius for ${ }^{11} \mathrm{Li}$ is now consistent with theoretical three-body models with a significant $1 s$-wave intruder state component, which reproduce breakup momentum distributions, but were hitherto thought to be at variance with cross section data. Our revised radius for ${ }^{11} \mathrm{Be}$ is also consistent with two-body models which include core excitation and reorientation effects.

The increased transparency of the few-body structures presented here is quite general, has implications for the deduced radii of all such exotic systems, and suggests that a careful reexamination of all such data is necessary. The particular importance of these effects in extended threebody halo systems is exciting. In the case of ${ }^{11} \mathrm{Li}$ we show this to be of importance in elucidating their structure and in bringing consistency between calculations and data for both breakup momentum distributions and reaction cross sections.
The financial support of the United Kingdom Engineering and Physical Sciences Research Council (EPSRC) in the form of Grants No. GR/J95867 and No. GR/K33026 is gratefully acknowledged. We would like to thank Dr. Filomena Nunes and Dr. Ian Thompson for providing two- and three-body wave functions for the ${ }^{11} \mathrm{Be}$ and ${ }^{11} \mathrm{Li}$ systems and Matthew Bush for providing elements of the static density Glauber model code used here. We thank Professor Ron Johnson for very useful comments and discussions resulting from an earlier draft of this Letter.

[1] I. Tanihata et al., Phys. Rev. Lett. 55, 2676 (1985).

[2] I. Tanihata et al., Phys. Lett. 160B, 380 (1985).

[3] I. Tanihata, T. Kobayashi, O. Yamakawa, S. Shimoura, K. Ekuni, K. Sugimoto, N. Takahashi, T. Shimoda, and H. Sato, Phys. Lett. B 206, 592 (1988).

[4] R. J. Glauber, in Lectures in Theoretical Physics, edited by W.E. Brittin (Interscience, New York, 1959), Vol. 1, p. 315.

[5] W. Czyz and L. C. Maximon, Ann. Phys. (N.Y.) 52, 59 (1969).

[6] The simplified Glauber theory used [3,5] is often called the optical limit, a term used in a number of contexts in the literature. We refer to this approximation as the static density limit in the present work.

[7] L. V. Chulkov, B. V. Danilin, V.D. Efros, A. A. Korshenninikov, and M. V. Zhukov, Europhys. Lett. 8, 245 (1989).

[8] S. Kox et al., Phys. Rev. C 35, 1678 (1987).

[9] I. J. Thompson (private communication).

[10] K. Yabana, Y. Ogawa, and Y. Suzuki, Phys. Rev. C 45, 2909 (1992); Nucl. Phys. A539, 295 (1992).

[11] J. S. Al-Khalili, I. J. Thompson, and J. A. Tostevin, Nucl. Phys. A581, 331 (1995).

[12] H. Nishioka and R. C. Johnson, Phys. Rev. C 22, 2457 (1980); J. Phys. G 8, 39 (1982).

[13] N. Takigawa, M. Ueda, M. Kuratani, and H. Sagawa, Phys. Lett. B 288, 244 (1992).

[14] S. K. Charagi and S. K. Gupta, Phys. Rev. C 41, 1610 (1990).

[15] Table I of Y. Ogawa, K. Yabana, and Y. Suzuki, Nucl. Phys. A543, 722 (1992).

[16] W. Bauhoff, At. Data Nucl. Data Tables 35, 429 (1986).

[17] T. Kobayashi et al., Phys. Lett. B 232, 51 (1989).

[18] I. J. Thompson and M. V. Zhukov, Phys. Rev. C 49, 1904 (1994).

[19] J. M. Bang and I. J. Thompson, Phys. Lett. B 270, 201 (1992).

[20] L. Johannsen, A. S. Jensen, and P. G. Hansen, Phys. Lett. B 244, 357 (1990).

[21] F. M. Nunes, I. J. Thompson, and R. C. Johnson, Nucl. Phys. A596, 171 (1996).

[22] I. Tanihata (private communication).

[23] B. Buck and A. Pilt, Nucl. Phys. A280, 133 (1977). 